\title{
The effects of hearing loss on neural processing and plasticity
}

\author{
Arthur Wingfield ${ }^{1 *}$ and Jonathan E. Peelle ${ }^{2 *}$ \\ 1 Volen National Center for Complex Systems, Brandeis University, Waltham, MA, USA, ${ }^{2}$ Department of Otolaryngology, \\ Washington University in St. Louis, St. Louis, MO, USA
}

Keywords: hearing loss, auditory cortex, cognition, aging, listening effort

Hearing loss-ranging from mild to severe-afflicts large numbers of individuals of all ages. It is estimated that $40-50 \%$ of adults over the age of 65 years have some degree of significant hearing loss, with this figure rising to $83 \%$ of those over the age of 70 (Cruickshanks et al., 1998). This makes hearing loss the third most prevalent chronic medical condition among older adults after arthritis and hypertension (Lethbridge-Cejku et al., 2004). Recent years have seen increasing appreciation for the downstream consequences of reduced hearing acuity, even when perception itself has been successful. In the case of speech, these consequences include negative effects of perceptual effort on encoding what has been heard in memory (Rabbitt, 1991; Surprenant, 1999; Pichora-Fuller, 2003; McCoy et al., 2005; Cousins et al., 2014) and comprehension of sentences whose processing is resource-demanding because of complex syntax (Wingfield et al., 2006). Beyond these short-term effects, there also appear to be small but statistically significant correlations between hearing acuity and the appearance of all-cause dementia (Gates et al., 2011; Lin et al., 2011b) and performance on standardized cognitive tests in non-demented individuals (Lin et al., 2011a). Strikingly, the relationship between hearing acuity and cognitive ability holds even when adjusted for sex, age, education, diabetes, smoking history, and hypertension (Lin, 2011; Lin et al., 2011a; Humes et al., 2013a).

The effects of impaired hearing thus goes beyond difficulty in speech recognition. Speech comprehension in the face of mild-to-moderate hearing loss modifies patterns of neural activation in BOLD imaging, and analyses of structural MRI images have shown that poor hearing acuity is associated with reduced gray matter volume in auditory cortex (Peelle et al., 2011; Eckert et al., 2012; Lin et al., 2014). Findings such as these indicate a biological link between sensory stimulation and cortical integrity, consistent with animal models demonstrating neural reorganization when sensory input is disrupted. In humans, these effects on auditory cortex may have cascading influences throughout the hierarchical set of regions involved in speech processing (Davis and Johnsrude, 2003; Rauschecker and Scott, 2009; Peelle et al., 2010).

Understanding sensory-cognitive interactions represents an important research challenge, especially when changes in hearing acuity are compounded by declines in working memory resources and executive function that often occur in adult aging. One must also note claims of an increase in hearing loss among young adults (Shargorodsky et al., 2010), many of whom remain unaware of their hearing loss and the consequences of perceptual effort on cognitive performance (Widen et al., 2009; Le Prell et al., 2011). At the level of remediation, surgically placed cochlear implants have seen increasing use, to include use with older adults, when hearing acuity has declined to a point where standard hearing aids no longer yield significant benefit (Dillon et al., 2013). This emerging technology will call increasingly on the translational potential of basic research in auditory physiology currently active in human and animal studies.

This research topic presents a collection of original articles that explore the cognitive and neural consequences of hearing loss, including basic processes carried out in the auditory periphery, computations in subcortical nuclei and primary auditory cortex, and higher-level processes such as those involved in human speech perception. Together, these 
articles form a compelling body of work demonstrating numerous ways in which brain structure, neural function, and behavior are impacted by hearing loss.

We begin with seven review and theory articles. Rönnberg and coauthors offer a timely update of the Ease of Language Understanding (ELU) model in which they stress the importance of working memory for online spoken language processing, especially under poor listening conditions (Rönnberg et al., 2013). Heald and Nusbaum (2014) continue this theme, arguing that even early-stage speech recognition is an attentionallyguided active process and not as automatic as some have suggested. Review articles by Guediche et al. (2014) and by Keating and King (2013) stress the flexibility in the perceptual system that allows for adaptation to auditory perturbations. Eggermont (2013) and by Butler and Lomber (2013) focus primarily on animal models to explore effects of experience on auditory processing, while Bharadwaj et al. (2014) review human and animals studies demonstrating that precision in temporal coding may be poor even when hearing thresholds are normal. Taken together, these papers emphasize the view that auditory detection thresholds give only a limited picture of auditory and auditory-cortical processing.

Additional evidence bearing on plasticity and development appears in six research articles using animal models. Gay et al. (2014) and Kang et al. (2014) explore mechanisms underlying interactions between early conductive hearing loss and effects on detection tasks in adulthood, while Kamal et al. (2013) focus on impact and reversibility of noise exposure effects in auditory cortex. Huetz et al. (2014) examine functional modification to cortical cells in response to moderate hearing loss. Henry et al. (2014) report effects of noise-induced sensorineural hearing loss on complex temporal coding, and Kral et al. (2013) examine the implications of hemisphere asymmetries in cortical adaptation to unilateral hearing loss in development.

Studies in human listeners reveal many of the same aspects of plasticity in the perceptual system as seen in animal models. Avivi-Reich et al. (2014) illustrate the dynamic interaction between bottom-up input and top-down cognitive factors when older adults are challenged by listening to a target speaker in a background of multiple speakers and when listening in a second language. Mishra et al. (2013) continue this theme with an emphasis on the role of selective attention when listening to speech in noise. Humes et al. (2013b) examine individual difference factors that influence successful speech comprehension

\section{References}

Alain, C. (2014). Effects of age-related hearing loss and background noise on neuromagnetic activity from auditory cortex. Front. Syst. Neurosci. 8:8. doi: 10.3389/fnsys.2014.00008

Anderson, S., White-Schwoch, T., Choi, H. J., and Kraus, N. (2013). Training changes processing of speech cues in older adults with hearing loss. Front. Syst. Neurosci. 7:97. doi: 10.3389/fnsys.2013.00097

Avivi-Reich, M., Daneman, M., and Schneider, B. A. (2014). How age and linguistic competence alter the interplay of perceptual and cognitive factors when listening to conversations in a noisy environment. Front. Syst. Neurosci. 8:21. doi: $10.3389 /$ fnsys.2014.00021 beyond peripheral hearing acuity. The value of in-depth studies of a single individual is illustrated by Firszt et al. (2013) who report neural and performance changes in an adult patient following successful surgery for a congenital unilateral hearing loss. Anderson et al. (2013) offer additional evidence bearing on plasticity in the sensory-cognitive system in a study of compensatory training through directed attention in hearing impaired older adults. McGettigan et al. (2014) address learning-related changes in speech recognition using noise-vocoded speech to simulate the acoustic input available from a cochlear implant. Finally, Ihlefeld et al. (2014) focus their research article on factors relating to cochlear implant recipients' decrements in the use of interaural time differences for localizing sound sources in space.

Considerable advances have been made using a number of human brain imaging techniques, as illustrated by a final eight articles in this collection that have examined effects of hearing loss using diffusion tensor imaging (DTI) to assess white matter integrity (Rachakonda et al., 2014), functional MRI to reveal patterns of neural reorganization and compensatory cognitive control with hearing loss and aging (Erb and Obleser, 2013; Husain et al., 2014), patterns of neural responses using electroencephalograph (EEG) recordings from scalp electrodes (Becker et al., 2013; Campbell and Sharma, 2013; Catz and Noreña, 2013; Tremblay et al., 2014) and magnetoencephalography (MEG) to examine contributory effects of reduced inhibitory control in older adults with hearing impairment (Alain, 2014).

Together, these collected articles reflect a valuable sample of current approaches to our understanding of the effects of hearing loss on neural and perceptual processing. A theme that emerges from both the human and animal studies in this collection is that of an adaptive plasticity in the sensory, perceptual and cognitive systems that regulates performance in the face of often seriously degraded input. Challenges for future research include better understanding the link between neural consequences of hearing loss and other modifications of acoustic input (Van Engen and Peelle, 2014) and a more direct linking of hearing ability, brain structure, neural function, and behavior.

\section{Acknowledgments}

The preparation of this manuscript was aided by NIH grants R01AG019714 and R01AG03890 from the National Institute on Aging and The Dana Foundation. 
Campbell, J., and Sharma, A. (2013). Compensatory changes in cortical resource allocation in adults with hearing loss. Front. Syst. Neurosci. 7:71. doi: $10.3389 /$ fnsys.2013.00071

Catz, N., and Noreña, A. (2013). Enhanced representation of spectral contrasts in the primary auditory cortex. Front. Syst. Neurosci. 7:21. doi: 10.3389/fnsys.2013.00021

Cousins, K. A. Q., Dar, H., Wingfield, A., and Miller, P. (2014). Acoustic masking disrupts time-dependent mechanisms of memory encoding in word-list recall. Mem. Cogn. 42, 622-638. doi: 10.3758/s13421-013-0377-7

Cruickshanks, K. J., Wiley, T. L., Tweed, T. S., Klein, B. E., Klein, R., MaresPerlman, J. A., et al. (1998). Prevalence of hearing loss in older adults in Beaver Dam, Wisconsin: the epidemiology of hearing loss study. Am. J. Epidemiol. 148, 879-886. doi: 10.1093/oxfordjournals.aje.a009713

Davis, M. H., and Johnsrude, I. S. (2003). Hierarchical processing in spoken language comprehension. J. Neurosci. 23, 3423-3431.

Dillon, M. T., Buss, E., Adunka, M. C., King, E. R., Pillsbury, H. C., Adunka, O. F., et al. (2013). Long-term speech perception in elderly cochlear implant users. JAMA Otolaryngol. Head Neck Surg. 139, 279-283. doi: 10.1001/jamaoto.2013.1814

Eckert, M. A., Cute, S. L., Vaden, K. I. Jr., Kuchinsky, S. E., and Dubno, J. R. (2012). Auditory cortex signs of age-related hearing loss. J. Assoc. Res. Otolaryngol. 13, 703-713. doi: 10.1007/s10162-012-0332-5

Eggermont, J. J. (2013). On the similarities and differences of non-traumatic sound exposure during the critical period and in adulthood. Front. Syst. Neurosci. 7:12. doi: $10.3389 /$ fnsys. 2013.00012

Erb, J., and Obleser, J. (2013). Upregulation of cognitive control networks in older adults' speech comprehension. Front. Syst. Neurosci. 7:116. doi: $10.3389 /$ fnsys.2013.00116

Firszt, J. B., Reeder, R. M., Holden, T. A., Burton, H., and Chole, R. A. (2013). Changes in auditory perceptions and cortex resulting from hearing recovery after extended congenital unilateral hearing loss. Front. Syst. Neurosci. 7:108. doi: $10.3389 /$ fnsys. 2013.00108

Gates, G. A., Anderson, M. L., McCurry, S. M., Feeney, M. P., and Larson, E. B. (2011). Central auditory dysfunction as a harbinger of Alzheimer dementia. Arch. Orolaryngol. 137, 390-395. doi: 10.1001/archoto.2011.28

Gay, J. D., Voytenko, S. V., Galazyuk, A. V., and Rosen, M. J. (2014). Developmental hearing loss impairs signal detection in noise: putative central mechanisms. Front. Syst. Neurosci. 8:162. doi: 10.3389/fnsys.2014.00162

Guediche, S., Blumstein, S., Fiez, J. A., and Holt, L. L. (2014). Speech perception under adverse conditions: insights from behavioral, computational and neuroscience research. Front. Syst. Neurosci. 7:126. doi: 10.3389/fnsys.2013.00126

Heald, S., and Nusbaum, H. C. (2014). Speech perception as an active cognitive process. Front. Syst. Neurosci. 8:35. doi: 10.3389/fnsys.2014.00035

Henry, K. S., Kale, S., and Heinz, M. G. (2014). Noise-induced hearing loss increases the temporal precision of complex envelope coding by auditory-nerve fibers. Front. Syst. Neurosci. 8:20. doi: 10.3389/fnsys.2014.00020

Huetz, C., Guedin, M., and Edeline, J.-M. (2014). Neural correlates of moderate hearing loss: time course of response changes in the primary auditory cortex of awake guinea-pigs. Front. Syst. Neurosci. 8:65. doi: 10.3389/fnsys.2014. 00065

Humes, L. E., Busey, T. A., Craig, J., and Kewley-Port, D. (2013a). Are agerelated changes in cognitive function driven by age-related changes in sensory processing? Atten. Percept. Psychophys. 75, 508-524. doi: 10.3758/s13414-0120406-9

Humes, L. E., Kidd, G. R., and Lentz, J. J. (2013b). Auditory and cognitive factors underlying individual differences in aided speech-understanding among older adults. Front. Syst. Neurosci. 7:55. doi: 10.3389/fnsys.2013.00055

Husain, F. T., Carpenter-Thompson, J. R., and Schmidt, S. A. (2014). The effect of mild-to-moderate hearing loss on auditory and emotion processing networks. Front. Syst. Neurosci. 8:10. doi: 10.3389/fnsys.2014.00010

Ihlefeld, A., Kan, A., and Litovsky, R. Y. (2014). Across-frequency combination of interaural time difference in bilateral cochlear implant listeners. Front. Syst. Neurosci. 8:22. doi: 10.3389/fnsys.2014.00022

Kamal, B. S., Holman, C., and de Villers-Sidani, E. (2013). Shaping the aging brain: role of auditory input patterns in the emergence of auditory cortical impairments. Front. Syst. Neurosci. 7:52. doi: 10.3389/fnsys.2013. 00052
Kang, R., Sarro, E., and Sanes, D. (2014). Auditory training during development mitigates a hearing loss-induced perceptual deficit. Front. Syst. Neurosci. 8:49. doi: $10.3389 /$ fnsys.2014.00049

Keating, P., and King, A. J. (2013). Developmental plasticity of spatial hearing following asymmetric hearing loss: context-dependent cue integration and its clinical implications. Front. Syst. Neurosci. 7:123. doi: 10.3389/fnsys.2013.00123

Kral, A., Heid, S., Hubka, P., and Tillein, J. (2013). Unilateral hearing during development: hemispheric specificity in plastic reorganizations. Front. Syst. Neurosci. 7:93. doi: $10.3389 /$ fnsys.2013.00093

Le Prell, C. G., Hensley, B. N., Campbell, K. C. M., Hall, J. W., and Guire, K. (2011). Evidence of hearing loss in a 'normally-hearing' college-student population. Int. J. Audiol. 50, S21-S31. doi: 10.3109/14992027.2010.540722

Lethbridge-Cejku, M., Schiller, J. S., and Bernadel, L. (2004). Summary health statistics for U.S. adults: national health inverview survey, 2002. Vital Health Stat. 10, 1-151.

Lin, F. R. (2011). Hearing loss and cognition among older adults in the United States. J. Gerontol. A Biol. Sci. Med. Sci. 66A, 1131-1136. doi: 10.1093/gerona/glr115

Lin, F. R., Ferrucci, L., An, Y., Goh, J. O., Doshi, J., Metter, E. J., et al. (2014). Association of hearing impairment with brain volume changes in older adults. Neuroimage 90, 84-92. doi: 10.1016/j.neuroimage.2013.12.059

Lin, F. R., Ferrucci, L., Metter, E. J., An, Y., Zonderman, A. B., and Resnick, S. M. (2011a). Hearing loss and cognition in the baltimore longitudinal study of aging. Neuropsychology 25, 763-770. doi: 10.1037/a0024238

Lin, F. R., Metter, J., O’Brien, R. J., Resnick, S. M., Zonderman, A. B., and Ferrucci, L. (2011b). Hearing loss and incident dementia. Arch. Neurol. 68, 214-220. doi: 10.1001/archneurol.2010.362

McCoy, S. L., Tun, P. A., Cox, L. C., Colangelo, M., Stewart, R., and Wingfield, A. (2005). Hearing loss and perceptual effort: downstream effects on older adults' memory for speech. Q. J. Exp. Psychol. 58, 22-33. doi: 10.1080/02724980443000151

McGettigan, C., Rosen, S., and Scott, S. K. (2014). Lexico-semantic and acoustic-phonetic processes in the perception of noise-vocoded speech: implications for cochlear implantation. Front. Syst. Neurosci. 8:18. doi: $10.3389 /$ fnsys.2014.00018

Mishra, S., Lunner, T., Stenfelt, S., Rönnberg, J., and Rudner, M. (2013). Seeing the talker's face supports executive processing of speech in steady state noise. Front. Syst. Neurosci. 7:96. doi: 10.3389/fnsys.2013.00096

Peelle, J. E., Johnsrude, I. S., and Davis, M. H. (2010). Hierarchical processing for speech in human auditory cortex and beyond. Front. Hum. Neurosci. 4:51. doi: 10.3389/fnhum.2010.00051

Peelle, J. E., Troiani, V., Grossman, M., and Wingfield, A. (2011). Hearing loss in older adults affects neural systems supporting speech comprehension. J. Neurosci. 31, 12638-12643. doi: 10.1523/JNEUROSCI.2559-11.2011

Pichora-Fuller, M. K. (2003). Cognitive aging and auditory information processing. Int. J. Audiol. 42, 2S26-2S32. doi: 10.3109/14992020309074641

Rabbitt, P. M. A. (1991). Mild hearing loss can cause apparent memory failures which increase with age and reduce with IQ. Acta Otolaryngolica Suppl. 476, 167-176. discussion: 176.

Rachakonda, T., Shimony, J. S., Coalson, R. S., and Lieu, J. E. C. (2014). Diffusion tensor imaging in children with unilateral hearing loss: a pilot study. Front. Syst. Neurosci. 8:87. doi: 10.3389/fnsys.2014.00087

Rauschecker, J. P., and Scott, S. K. (2009). Maps and streams in the auditory cortex: nonhuman primates illuminate human speech processing. Nat. Neurosci. 12, 718-724. doi: 10.1038/nn.2331

Rönnberg, J., Lunner, T., Zekveld, A., Sörqvist, P., Danielsson, H., Lyxell, B., et al. (2013). The ease of language understanding (ELU) model: theoretical, empirical, and clinical advances. Front. Syst. Neurosci. 7:31. doi: $10.3389 /$ fnsys.2013.00031

Shargorodsky, J., Curhan, S. G., Curhan, G. C., and Eavey, R. (2010). Change in prevalence of hearing loss in US adolescents. J. Am. Med. Assoc. 304, 772-778. doi: 10.1001/jama.2010.1124

Surprenant, A. M. (1999). The effect of noise on memory for spoken syllables. Int. J. Psychol. 34, 328-333. doi: 10.1080/002075999399648

Tremblay, K., Ross, B., Inoue, K., McClannahan, K., and Collet, G. (2014). Is the auditory evoked P2 response a biomarker of learning? Front. Syst. Neurosci. 8:28. doi: $10.3389 /$ fnsys.2014.00028 
Van Engen, K. J., and Peelle, J. E. (2014). Listening effort and accented speech. Front. Hum. Neurosci. 8:577. doi: 10.3389/fnhum.2014.00577

Widen, S. E., Holmes, A. E., Johnson, T., Bohlin, M., and Erlandsson, S. I. (2009). Hearing, use of hearing protection, and attitudes towards noise among young American adults. Int. J. Audiol. 48, 537-545. doi: 10.1080/14992020902 894541

Wingfield, A., McCoy, S. L., Peelle, J. E., Tun, P. A., and Cox, C. (2006). Effects of adult aging and hearing loss on comprehension of rapid speech varying in syntactic complexity. J. Am. Acad. Audiol. 17, 487-497. doi: 10.3766/ jaaa.17.7.4
Conflict of Interest Statement: The authors declare that the research was conducted in the absence of any commercial or financial relationships that could be construed as a potential conflict of interest.

Copyright $\odot 2015$ Wingfield and Peelle. This is an open-access article distributed under the terms of the Creative Commons Attribution License (CC BY). The use, distribution or reproduction in other forums is permitted, provided the original author(s) or licensor are credited and that the original publication in this journal is cited, in accordance with accepted academic practice. No use, distribution or reproduction is permitted which does not comply with these terms. 